\title{
UNICITY OF MEROMORPHIC FUNCTIONS CONCERNING DIFFERENTIAL EQUATION
}

\author{
SUBHAS S. BHOOSNURMATH, MILIND N. KULKARNI, VAISHALI PRABHU \\ |||
}

\begin{abstract}
In this paper,we investigate the uniqueness of meromorphic function satisfying a differential equation. Our result improves some known results.
\end{abstract}

\section{Introduction}

In this paper, the term 'meromorphic' means meromorphic in the whole complex plane.It is assumed that the reader is familiar with notations of Nevanlinna Theory as in [2]. We denote by $S(r, f)$ any function satisfying $S(r, f)=o(T(r, f))$ as $r \rightarrow \infty$, possibly outside the set of finite measure. We say $a(z)$ is a small meromorphic function of $f$ if $T(r, a(z))=S(r, f)$.

Subhas. S. Bhoosnurmath and K. S. L. N. Prasad [1] have proved the following Theorem on uniqueness of meromorphic functions sharing a small meromorphic function.

Theorem $\boldsymbol{A}$. Let $f$ be a non-constant transcendental meromorphic function with $N(r, f)+N\left(r, \frac{1}{f}\right)=S(r, f)$ Let ' $a^{\prime}$ be a small meromorphic function of $f$ i.e.

$$
T(r, a)=S(r, f) .
$$

If $f$ satisfies the equation,

$$
k f^{\prime}-f-(k-1) a=0
$$

for $k \neq 0$, then $f=f^{\prime}$.

\section{We require the following definitions}

By a Monomial in $f$ we mean an expression of the type

$$
M_{j}(f)=a_{j}(z)(f(z))^{n_{o j}}\left(f^{\prime}(z)\right)^{n_{1 j}} \cdots\left(f^{(k)}(z)\right)^{n_{k j}}
$$

where $n_{0 j}, n_{1 j}, \ldots, n_{k j}$ are non-negative integers. We define $\bar{d}\left(M_{j}\right)=\sum_{i=o}^{k} n_{i j}$ as the degree of $M_{j}(f)$ and $\Gamma\left(M_{j}\right)=\sum_{i=o}^{k}(i+1) n_{i j}$ as the weight of $M_{j}(f)$.

Received December 04, 2005; revised March 09, 2006.

Key words and phrases. Meromorphic functions, shared value, differential polynomial. 
Differential polynomial in $f$ is a finite sum of such monomials i.e.

$$
P(f)=\sum_{j=1}^{s} a_{j}(z) M_{j}(f) .
$$

We define $\bar{d}(P)=\max _{1 \leq j \leq s}\left\{\bar{d}\left(M_{j}\right)\right\}$ as the degree, $\underline{d}(P)=\min _{1 \leq j \leq s}\left\{\bar{d}\left(M_{j}\right)\right\}$ as the lower degree and $\Gamma(P)=\max _{1 \leq j \leq s}\left\{\Gamma\left(M_{j}\right)\right\}$ as the weight of $P(f)$.

If $\bar{d}(P)=\underline{d}(P)=n, P(f)$ is a called homogeneous differential polynomial and inhomogeneous otherwise.

We shall improve Theorem A for a homogeneous differential polynomial in $f$ as follows.

\section{Statement of main Theorem}

Theorem 1. Let $f(z)$ be a non-constant transcendental meromorphic function with $N(r, f)+N\left(r, \frac{1}{f}\right)=S(r, f)$. Let $P(f)$ be a homogeneous differential polynomial in f.Let ' $a$ ' be a small meromorphic function of $f$ i.e.

$$
T(r, a(z))=S(r, f) .
$$

If $f$ satisfies the equation

$$
k P(f)-f-(k-1) a=0 \text { for } \quad k \neq 0
$$

Then, $f \equiv P(f)$.

We require the following lemmas to prove our results.

Lemma 1.([3]) Let $f_{1}$ and $f_{2}$ be two non-constant meromorphic functions and $\alpha_{1} \neq o$, $\alpha_{2} \neq o$ be two small meromorphic functions satisfying,

$$
T\left(r, \alpha_{i}\right)=S(r, f) \text { for } i=1,2
$$

where,

$$
T(r, f)=\max \left\{T\left(r, f_{1}\right), T\left(r, f_{2}\right)\right\} .
$$

If $\alpha_{1} f_{1}+\alpha_{2} f_{2} \equiv 1$, then

$$
\begin{gathered}
T\left(r, f_{1}\right)<\bar{N}\left(r, \frac{1}{f_{1}}\right)+\bar{N}\left(r, \frac{1}{f_{2}}+\bar{N}\left(r, f_{1}\right)+o\{T(r, f)\},\right. \\
T\left(r, f_{2}\right)<\bar{N}\left(r, \frac{1}{f_{2}}\right)+\bar{N}\left(r, \frac{1}{f_{1}}\right)+\bar{N}\left(r, f_{2}\right)+o\{T(r, f)\} .
\end{gathered}
$$


Lemma 2. If $f$ is a non-constant transcendental meromorphic function and if $P(f)$ is a homogeneous differential polynomial in $f$ of degree $n$, then

$$
N\left(r, \frac{P(f)}{f^{n}}\right) \leq m n\left[\bar{N}(r, f)+\bar{N}\left(r, \frac{1}{f}\right)\right]+S(r, f),
$$

where $m$ is the order of highest order derivative occuring in $P(f)$.

Proof of Lemma 2. Since $m$ is the order of the highest derivative $f^{(m)}$ occuring in $P$. Then clearly a zero or a pole of $f$ which is not a pole of any co-efficient $a(z)$ of $P$, is a pole of $\frac{P}{f^{n}}$ of degree $m n$ atmost. Hence we have,

$$
N\left(r, \frac{P}{f^{n}}\right) \leq m n\left[\bar{N}(r, f)+\bar{N}\left(r, \frac{1}{f}\right)\right]+S(r, f) .
$$

Lemma 3.([4]) If $f$ is a non-constant trancsendental meromorphic function and if $P(f)$ is a homogeneous differential polynomial in $f$ of degree $n$, then

$$
m\left(r, \frac{P(f)}{f^{n}}\right)=S(r, f) .
$$

Lemma 4. If $f$ is a non-constant transcendental meromorphic function and if $P(f)$ is a homogeneous differential polynomial in $f$ of degree $n$ then,

$$
N\left(r, \frac{1}{P(f)}\right) \leq m n\left[\bar{N}(r, f)+\bar{N}\left(r, \frac{1}{f}\right)\right]+n N\left(r, \frac{1}{f}\right)+S(r, f),
$$

where $m$ is the order of highest order derivative occuring in $P(f)$.

Proof of Lemma 4. Consider,

$$
\begin{aligned}
N\left(r, \frac{1}{P(f)}\right) & \leq N\left(r, \frac{f^{n}}{P(f)}\right)+N\left(r, \frac{1}{f^{n}}\right) \\
& \leq T\left(r, \frac{f^{n}}{P(f)}\right)+n N\left(r, \frac{1}{f}\right)+S(r, f) \\
& =m\left(r, \frac{P(f)}{f^{n}}\right)+N\left(r, \frac{P(f)}{f^{n}}\right)+n N\left(r, \frac{1}{f}\right)+O(1) \quad \text { by Lemma } 2 \text { and } 4, \\
& \leq S(r, f)+m n\left[\bar{N}(r, f)+\bar{N}\left(r, \frac{1}{f}\right)\right]+n N\left(r, \frac{1}{f}\right)+O(1) \\
N\left(r, \frac{1}{P(f)}\right) & \leq m n\left[\bar{N}(r, f)+\bar{N}\left(r, \frac{1}{f}\right)\right]+n N\left(r, \frac{1}{f}\right)+S(r, f) .
\end{aligned}
$$

Proof of Theorem 1. We have,

$$
\begin{aligned}
k P(f)-f-(k-1) a & =0 \\
\Rightarrow k(P(f)-a) & =f-a \\
\Rightarrow \frac{f-a}{P(f)-a} & =k \text { where } k \neq 0
\end{aligned}
$$


Put $f_{1}=\frac{1}{a} f, f_{2}=k, f_{3}=\frac{-k}{a} P(f)$ where $(a \neq 0)$, so that $f_{1}+f_{2}+f_{3} \equiv 1$.

If $k \neq 1$, we get,

$$
\frac{1}{a(1-k)} f-\frac{k}{a(1-k)} P(f) \equiv 1 \text {. }
$$

By lemma 1. we have,

$$
\begin{gathered}
T(r, f)<\bar{N}\left(r, \frac{1}{P(f)}\right)+\bar{N}\left(r, \frac{1}{f}\right)+\bar{N}(r, f)+S(r, f), \\
T(r, P(f))<\bar{N}\left(r, \frac{1}{P(f)}\right)+\bar{N}\left(r, \frac{1}{f}\right)+\bar{N}(r, P(f))+S(r, f) .
\end{gathered}
$$

Also we have,

$$
N(r, P(f)) \leq n N(r, f)+m n \bar{N}(r, f)+S(r, f),
$$

where $m$ is degree of highest order derivative occuring in it. Also by Lemma 4, we get

$$
N\left(r, \frac{1}{P(f)}\right) \leq m n\left[\bar{N}(r, f)+\bar{N}\left(r, \frac{1}{f}\right)\right]+n N\left(r, \frac{1}{f}\right)+S(r, f) .
$$

Therefore,using lemma 4, (2) and (3) can be written as

$$
\begin{aligned}
T(r, f) \leq & \bar{N}\left(r, \frac{1}{f}\right)+m n\left[\bar{N}(r, f)+\bar{N}\left(r, \frac{1}{f}\right)\right]+n N\left(r, \frac{1}{f}\right)+\bar{N}(r, f)+S(r, f), \\
T(r, P(f)) \leq & m n\left[\bar{N}(r, f)+\bar{N}\left(r, \frac{1}{f}\right)\right]+n N\left(r, \frac{1}{f}\right)+\bar{N}\left(r, \frac{1}{f}\right)+n N(r, f) \\
& +m n \bar{N}(r, f)+S(r, f) .
\end{aligned}
$$

Adding (4) and (5) we get,

$$
\begin{aligned}
T(r, f)+T(r, P(f)) \leq & 2 m n\left[\bar{N}(r, f)+\bar{N}\left(r, \frac{1}{f}\right)\right]+2 n N\left(r, \frac{1}{f}\right)+2 \bar{N}\left(r, \frac{1}{f}\right) \\
& +n N(r, f)+(m n+1) \bar{N}(r, f)+S(r, f) \\
\leq & 2(m n+n+1) N\left(r, \frac{1}{f}\right)+(3 m n+n+1) N(r, f)+S(r, f) \\
\leq & (3 m n+2 n+2)\left(N\left(r, \frac{1}{f}\right)+N(r, f)\right)+S(r, f)
\end{aligned}
$$

$\leq S(r, f)$ which is contradiction, as the above relation implies

$$
1 \leq \frac{S(r, f)}{T(r, f)+T(r, P(f))} \rightarrow 0, \quad \text { as } \quad r \rightarrow \infty
$$

possibly outside a set of finite measure. Hence, $k=1$ Therefore

$$
f \equiv P(f) .
$$




\section{Acknowledgements}

The authors thank the refrees for their valuable suggestions.

The second author thanks the University Grants Comission (U.G.C (India)) for the award of Teacher Fellowship under Faculty Improvement Programme.

\section{References}

[1] K. S. L. N. Prasad, The value Distribution of Differential Polynomials, Meromorphic matrix valued functions and related topics, Ph.D thesis submitted to Karnatak University Dharwad in 2002 .

[2] W. K. Hayman, Meromorphic Functions, Clarendon Press, Oxford, 1964.

[3] Indrajit Lahiri, Uniqueness of meromorphic functions sharing same 1 points, Bull. Korean Math. Society 35(1998), 375-385.

[4] H. S. Gopalkrishna and S. S. Bhoosnurmath, On deficiencies of Differential Polynomials, Karnataka University Journal of Science 28(1973), 329-335.

Department of Mathematics, Karnatak University Dharwad.

E-mail: ssbmath@yahoo.com

E-mail: meelind2000@yahoo.co.in

E-mail:vaishali23nov@yahoo.co.in 
